INPLASY

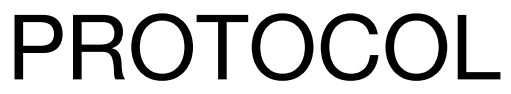

To cite: Gao et al. Association between tumor necrosis factor $a$ and uterine fibroids: a protocol of systematic review. Inplasy protocol 202070010. doi:

10.37766/inplasy2020.7.0010

Received: 03 July 2020

Published: 03 July 2020

Corresponding author: Li-nan Gao

hjws417129@sina.cn

Author Affiliation:

Affiliated Hospital of Jilin

Medical College

Support: JLPHHMMIP

(2019G010)

Review Stage at time of this submission: The review has not yet started.

\section{Association between tumor necrosis factor $a$ and uterine fibroids: a protocol of systematic review}

Gao, LN1; Ge, LG²; Zhu, MZ3; Yao, XX

Review question / Objective: Does tumor necrosis factor a (TNF-a) have association with uterine fibroids (UFs)?

Condition being studied: Tumor necrosis factor $a$; uterine fibroids.

Information sources: From inception to the present, electronic databases will be searched in Cochrane Library, PUBMED, EMBASE, Web of Science, WANGFANG, Chinese Biomedical Literature Database, and China National Knowledge Infrastructure. We will consider CCSs addressing the association between TNF- $a$ and UFs. The template of search strategy of PUBMED is summarized. Identical search strategies for other electronic databases will be modified. In addition, we will search ongoing studies in clinical registry trials, conference proceedings, and reference lists of relevant reviews.

INPLASY registration number: This protocol was registered with the International Platform of Registered Systematic Review and Meta-Analysis Protocols (INPLASY) on 03 July 2020 and was last updated on 03 July 2020 (registration number INPLASY202070010).

Conflicts of interest:

None.

\section{INTRODUCTION}

Review question / Objective: Does tumor necrosis factor a (TNF-a) have association with uterine fibroids (UFs)?

Condition being studied: Tumor necrosis factor $a$; uterine fibroids.

\section{METHODS}

Participant or population: Patients who were diagnosed as UFs, and normal healthy participants will be included.

Intervention: Patients who were diagnosed as UFs will be included in the experimental group. 
Comparator: Normal healthy participants will be considered in the control group.

Study designs to be included: All potential case-controlled studies (CCSs) exploring the association between TNF- $a$ and UFs will be considered.

Eligibility criteria: All potential CCSs exploring the association between TNF-a and UFs will be considered.

Information sources: From inception to the present, electronic databases will be searched in Cochrane Library, PUBMED, EMBASE, Web of Science, WANGFANG, Chinese Biomedical Literature Database, and China National Knowledge Infrastructure. We will consider CCSs addressing the association between TNF-a and UFs. The template of search strategy of PUBMED is summarized. Identical search strategies for other electronic databases will be modified. In addition, we will search ongoing studies in clinical registry trials, conference proceedings, and reference lists of relevant reviews.

Main outcome(s): We will assess the outcome indicators based on the studies concerning the association between TNF-a and UFs, such as gene and protein expression of TNF- $a$, proportion requiring hysterectomy, quality of life, and successful pregnancies.

Data management: Two researchers will independently collect data using standard data extraction form. The following information consists of basic information (study ID, publication time and source, first author, etc), characteristics of study (study setting, study methods, sample size, etc), intervention and control indexes, outcomes, following up information, results and findings. Any disagreement will be solved by discussion with another researcher.

Quality assessment / Risk of bias analysis: The quality of eligible studies will be assessed by two researchers using The Newcastle-Ottawa Scale. Any division will be solved by another researcher through consultation, and a consensus will be reached.

Strategy of data synthesis: We will perform RevMan 5.3 software to analyze statistical analysis. The weighted mean difference or standardized mean difference and 95\% confidence intervals (Cls), and risk ratio and $95 \%$ Cls will be estimated to present data synthesis outcome of continuous data and dichotomous data, respectively. Statistical heterogeneity will be checked by $I^{2}$ test, and a coarse guide for its explanation is as follows: $I^{2}<40 \%$ indicates that there might be minor heterogeneity, and we will use a fixed-effects model; $40 \% \leq I^{2}<75 \%$ means moderate heterogeneity; and we will employ a random-effects model; and $I^{2} \geq 75 \%$ means significant heterogeneity, and metaanalysis is deemed not to be performed. If $1^{2} \geq 40 \%$, the source of heterogeneity will be conducted using subgroup analysis and meta-regression test.

Subgroup analysis: Subgroup analysis and meta-regression test will be conducted according to the characteristics of the study participants, study quality, and sample size.

Sensibility analysis: Sensitivity analysis will be performed to examine the robustness of study findings by taking away low study quality.

\section{Country(ies) involved: China.}

Keywords: Uterine fibroids; tumor necrosis factor $\mathbf{a}$; association.

Contributions of each author:

Author 1 - Li-nan Gao.

Author 2 - Lian-gang Ge.

Author 3 - Ming-zhe Zhu.

Author 4 - Xin-xin Yao. 